\title{
Increased TRPM4 Activity in Cerebral Artery Myocytes Contributes to Cerebral Blood Flow Reduction After Subarachnoid Hemorrhage in Rats
}

\author{
Yi Gong ${ }^{1,2,3} \cdot$ Ming-yue Du ${ }^{1} \cdot$ Hua-lin Yu ${ }^{1} \cdot$ Zhi-yong Yang $^{1} \cdot$ Yu-jin Li ${ }^{4} \cdot$ Lei Zhou $^{5} \cdot$ Rong Mei $^{6} \cdot$ Li Yang $^{7} \cdot$ Fei Wang $^{1,2}$
}

Published online: 9 May 2019

(C) The American Society for Experimental NeuroTherapeutics, Inc. 2019

\begin{abstract}
Cerebral blood flow (CBF) reduction underlies unfavorable outcomes after subarachnoid hemorrhage (SAH). Transient receptor potential melastatin-4 (TRPM4) has a pivotal role in cerebral artery myogenic tone maintenance and CBF regulation under physiological conditions. However, the role of TRPM4 in CBF reduction after SAH is unclear. In this study, we aimed at testing whether TRPM4 would contribute to CBF reduction after SAH in vivo and determining underlying mechanisms. Rat SAH model was established by stereotaxic injection of autologous nonheparinized arterial blood at the suprasellar cistern. A TRPM4 blocker, 9-phenanthrol (9-Phe), was infused through an intraventricular catheter connected to a programmed subcutaneous pump to evaluate the contribution of TRPM4 to SAH outcomes. TRPM4 expression and translocation in cerebral artery myocytes were detected by immunoblotting. Macroscopic currents in cerebral artery myocytes were determined by whole-cell patch clamp. Myogenic tone of cerebral arteries was studied by pressurized myography. Cortical and global CBFs were measured via laser Doppler flowmetry and fluorescent microspheres, respectively. After SAH, TRPM4 translocation and macroscopic current density increased significantly. Furthermore, TRPM4 accounted for a greater proportion of myogenic tone after SAH, suggesting an upregulation of TRPM4 activity in response to SAH. Cortical and global CBFs were reduced after SAH, but were restored significantly by 9-Phe, implying that TRPM4 contributed to CBF reduction after SAH. Collectively, these discoveries show that increased TRPM4 activity has a pivotal role in CBF reduction after SAH, and provide a novel target for the management of cerebral perfusion dysfunction following $\mathrm{SAH}$.
\end{abstract}

Key Words Subarachnoid hemorrhage $\cdot$ transient receptor potential melastatin- $4 \cdot$ myogenic tone $\cdot$ cerebral vasospasm $\cdot$ cerebral blood flow.

Electronic supplementary material The online version of this article (https://doi.org/10.1007/s13311-019-00741-4) contains supplementary material, which is available to authorized users.

Fei Wang

neurosurgeonwf@aliyun.com

Yi Gong

gy19841013@163.com

1 Department of Neurosurgery, The First Affiliated Hospital of Kunming Medical University, 295 Xichang Road, Kunming 650032, China

2 Yunnan Key Laboratory of Laboratory Medicine, Kunming 650032, China

3 Department of Neurosurgery, The Third People's Hospital of Yunnan Province, Kunming 650011, China
4 Department of Anesthesiology, The First People's Hospital of Yunnan Province, Kunming 650032, China

5 The Key Laboratory of Stem Cell and Regenerative Medicine of Yunnan Province, Institute of Molecular and Clinical Medicine, Kunming Medical University, Kunming 650500, China

6 Department of Neurology, The First People's Hospital of Yunnan Province, Kunming 650500, China

7 Department of Anatomy, Histology and Embryology, Kunming Medical University, Kunming 650500, China 


\section{Introduction}

Subarachnoid hemorrhage (SAH) is typically caused by spontaneous breakage of a cerebral aneurysm situated at the bottom of the skull, followed by bleeding into the subarachnoid space [1]. It is a subtype of hemorrhagic stroke that is responsible for up to $10 \%$ of all stroke cases, accounting for $\sim 20 \%$ of cerebrovascularassociated deaths. Furthermore, $30 \%$ of patients who survives SAH are severely disabled [2]. Cerebral blood flow (CBF) reduction is the underlying cause of this unfavorable outcome and occurs in up to $70 \%$ of SAH patients [3]. The risk of CBF reduction, independent of elevated intracranial pressure, is primarily related to the severity of the initial bleeding. Multiple mechanisms are responsible for $\mathrm{CBF}$ reduction after $\mathrm{SAH}$, including large-vessel narrowing (vasospasm), microcirculation dysfunction, early brain injury (EBI), cortical spreading depolarization (CSD), microthrombosis, and neurovascular uncoupling [4]. Although CBF reduction is theoretically preventable, few pharmacological treatments are available, and unfortunately, they have yielded disappointing results. Therefore, understanding the mechanisms of CBF reduction after SAH is of paramount importance for the discovery of novel, effective targets for SAH treatment.

Enhanced contractility of cerebral arteries is believed to be one of the important underlying causes of vasospasm [5]. Previously, we showed that R-type calcium channels in cerebral artery myocytes contribute to CBF reduction after SAH via increased calcium influx and the subsequent activation of intracellular contractile proteins [6]. Cerebral artery myocytes show hypersensitivity to changes in intravascular pressure [7]; however, calcium channels in cerebral artery myocytes are not able to sense such changes [8] and the upstream pathways governing the role of calcium channels in vasospasm are not completely understood. Transient receptor potential melastatin-4 (TRPM4), permeable to monovalent ions and activated by extracellular calcium, is expressed in cerebral artery myocytes and activates calcium channels through membrane depolarization [9]. Additionally, TRPM4 is able to directly sense intravascular pressure [9] and exerts a pivotal role in the maintenance of cerebral artery myogenic tone and $\mathrm{CBF}$ regulation under physiological conditions $[10,11]$. However, the activity of TRPM4 and its role in $\mathrm{CBF}$ reduction after SAH have not been elucidated. In this study, we tested whether TRPM4 would contribute to CBF reduction after $\mathrm{SAH}$ in vivo and determined the involved mechanisms.

\section{Materials and Methods}

\section{Animals and Study Design}

Sprague-Dawley (SD) rats in both sexes $(n=120,300-350 \mathrm{~g})$, were obtained from the Experimental Animal Center of
Kunming Medical University (SCXK [Yunnan] 2011-0004). Rats were treated humanely following the Principles of Laboratory Animal Care established by the Kunming Medical University Committee of Medical Research, and the Guide for the Use of Laboratory Animals implemented by the Institute of Laboratory Animal Resources of Kunming Medical University. Forty-eight rats were randomly assigned to the following 2 groups using a random number table method for immunofluorescence, patch clamping, and pressurized myography: $\mathrm{SAH}$ $(n=24)$ and sham $(n=24)$, and then subgrouped for 3 time points (days 3, 5, and 7;n=8/group) within each group (Fig. 1a). Another 72 rats were randomly assigned to the following 4 groups by a random number table method for cortical and global CBF measurements: sham ( $n=18), \mathrm{SAH}(n=18)$, sham + 9-phenanthrol (9-Phe) $(n=18)$, and SAH + 9-Phe $(n=18)$, and then equally subgrouped for 3 time points (days 3,5 , and $7 ; n=$ 6/group) within each group (Fig. 1b). Animals were kept in a 12-h light/dark cycle with controlled temperature and humidity and accessed to food and water ad libitum.

\section{Rat SAH Model and Programmed Pump Implantation}

Rat SAH model was performed under the guidance of a stereotaxic apparatus as previously described [6]. Briefly, for the SAH and SAH + 9-Phe groups, $0.2 \mathrm{~mL}$ of autologous nonheparinized arterial blood was injected into suprasellar cistern, whereas the same volume of isotonic saline was injected for the sham group and the sham + 9-Phe group. Subsequently, a catheter was implanted into the right lateral cerebral ventricle for cortical and global CBF measurements using stereotaxic coordinates as follows: bregma, $-0.8 \mathrm{~mm}$; lateral, $1.4 \mathrm{~mm}$; and depth, $4 \mathrm{~mm}$ [11]. The catheter was then connected to a programmed pump (IPRECIO SMP-200 type, Durect Corporation, Cupertino, CA, USA), which was implanted subcutaneously at the back of the animal (Fig. 2b). Scalp incision was closed using a 1-0 nylon suture. Rats were then hydrated with $20 \mathrm{~mL}$ isotonic saline, s.c. [6]. For the Sham + 9-Phe and SAH + 9-Phe groups, the pump contained $900 \mu \mathrm{L}$ 9-Phe $(0.03 \mathrm{mM}$, Sigma, St. Louis, MO, USA) [12]. For the sham and SAH groups, the pump contained $900 \mu \mathrm{L}$ isotonic saline. Before implantation, the pump was programmed using iPRECIO management software (IMS-200, Durect Corporation). Each pump was set to continuously release 9-Phe or saline at a rate of $13 \mu \mathrm{L} / \mathrm{h}$. Immunofluorescence, patch clamp, pressurized myography, and CBF measurement were performed at 3 time points independently (days 3, 5, and 7) (Fig. 2a).

\section{Isolation of Cerebral Artery Myocytes}

Rats were killed via decapitation under deep anesthesia (pentobarbital, $60 \mathrm{mg} / \mathrm{kg}$, i.p.). Brains were harvested and immersed in cold $\left(4{ }^{\circ} \mathrm{C}\right)$ artificial cerebrospinal fluid $(\mathrm{aCSF})$ containing (in $\mathrm{mM}$ ) the following: $125 \mathrm{NaCl}, 3 \mathrm{KCl}, 18 \mathrm{NaHCO}_{3}, 1.25$ $\mathrm{NaH}_{2} \mathrm{PO}_{4}, 1 \mathrm{MgCl}_{2}, 2 \mathrm{CaCl}_{2}$, and 5 glucose (pH 7.3). Cerebral 
Fig. 1 Schematic diagrams showing experimental designs of the current study. Forty-eight rats were randomly divided into 2 groups for immunofluorescence, patch clamp, and pressurized myography (sham $(n=24), \mathrm{SAH}$ $(n=24))$ and were further subgrouped by time point (day 3 , 5, and 7) (a). Another 72 rats were randomly divided into the following 4 groups for cortical and global CBF measurements (sham $(n=18), \mathrm{SAH}(n=18)$, sham + 9-Phe $(n=18)$, and SAH +9 -Phe $(n=18))$ and were further subgrouped by time point (day 3 , day 5 , and day 7) (b) a

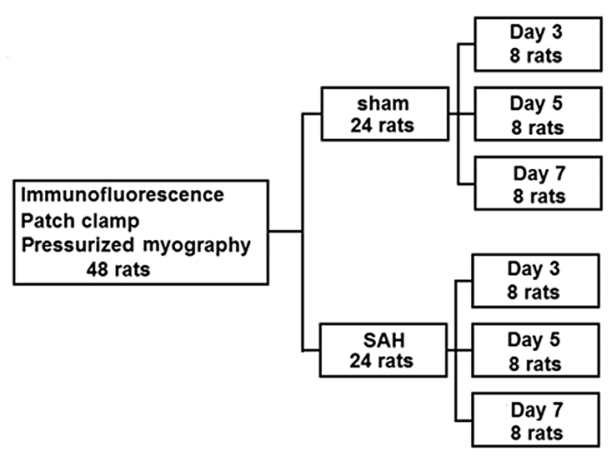

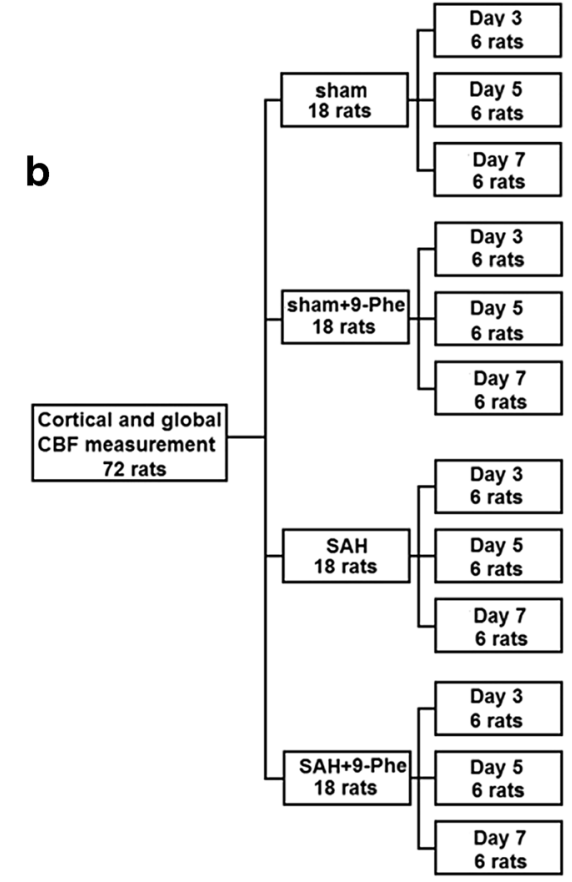

arteries (MCAs, ACAs, and PCAs) were separated from the brain and arachnoid membrane, followed by immersing in cold aCSF before cell isolation. Cerebral artery myocytes were isolated enzymatically as described previously [13]. Briefly, cerebral arteries were incubated in an isolation solution $(55 \mathrm{mM} \mathrm{NaCl}$, $5.6 \mathrm{mM} \mathrm{KCl}, 80 \mathrm{mM}$ sodium glutamate, $2 \mathrm{mM} \mathrm{MgCl}_{2}, 100 \mu \mathrm{M}$ $\mathrm{CaCl}_{2}, 10 \mathrm{mM}$ HEPES, and $10 \mathrm{mM}$ glucose, $\mathrm{pH}$ 7.3) supplemented with $0.3 \mathrm{mg} / \mathrm{mL}$ papain (Sigma) and $0.7 \mathrm{mg} / \mathrm{mL}$ dithioerythritol (Sigma) at $37{ }^{\circ} \mathrm{C}$ for $20 \mathrm{~min}$. Cerebral artery portions were then placed in an isolation solution consisting $0.7 \mathrm{mg} / \mathrm{mL}$ type $\mathrm{F}$ collagenase (Sigma) and $0.3 \mathrm{mg} / \mathrm{mL}$ type $\mathrm{H}$ collagenase (Sigma) and incubated at $37^{\circ} \mathrm{C}$ for another $20 \mathrm{~min}$.
Artery portions were washed 4 times after incubation with a cold isolation solution and then slowly triturated to yield individual myocytes. Myocytes were maintained in an isolation solution on ice for analysis within $6 \mathrm{~h}$.

\section{TRPM4 Immunofluorescence Staining}

Isolated cerebral artery myocytes were permitted to adher on glass slides for $30 \mathrm{~min}$ at $4{ }^{\circ} \mathrm{C}$. Myocytes were then fixed using $4 \%$ formaldehyde for $20 \mathrm{~min}$, followed by permeabilization in $0.5 \%$ Triton $\mathrm{X}-100$ for $25 \mathrm{~min}$ at room temperature. Subsequently, slides were blocked with $5 \%$ goat serum
Fig. 2 Diagrams showing experimental protocols. SAH model development and 9-Phe treatment time-lines (a). Image showing the location of subcutaneous pump (b) b
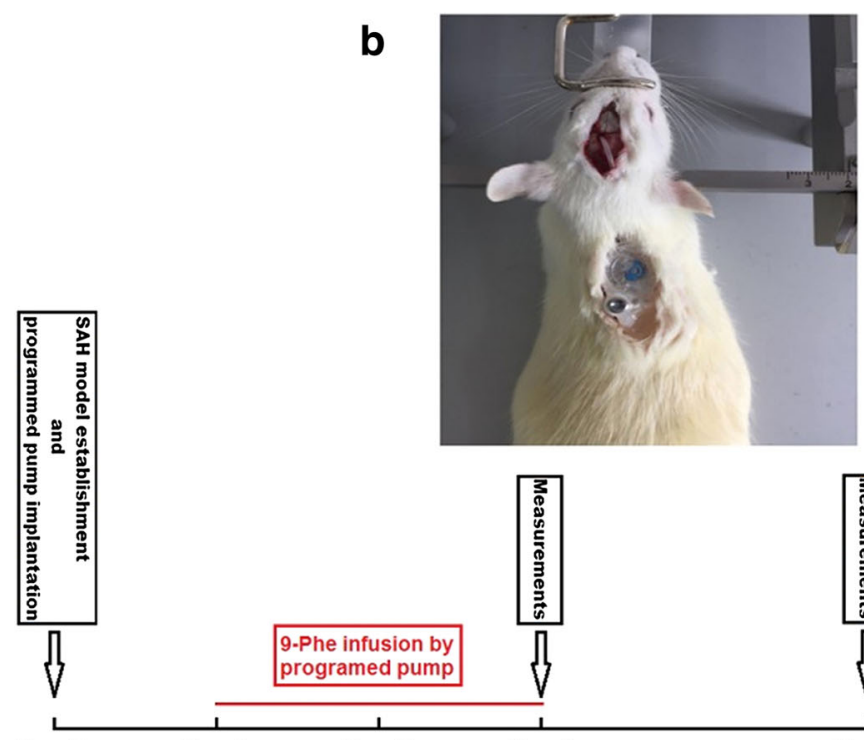

Day 0

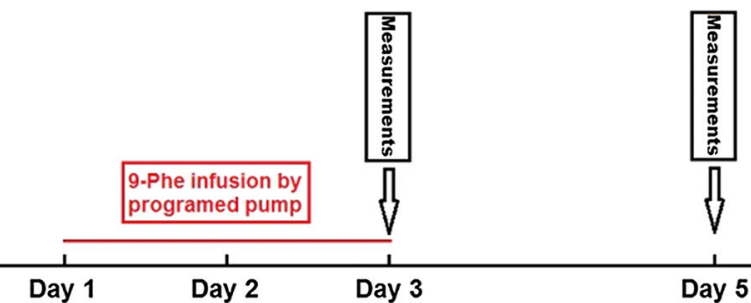

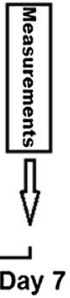


albumin for $35 \mathrm{~min}$ and hybridized with polyclonal rabbit anti-TRPM4 (1:50, Abcam, Cambridge, MA, USA) overnight at $4{ }^{\circ} \mathrm{C}$. After incubation, slides were rinsed with phosphatebuffered saline (PBS) and then hybridized with rat anti-rabbit monoclonal fluorescent secondary antibody (1:100, Abcam) at room temperature for $1 \mathrm{~h}$. Finally, cell nuclei were stained with DAPI (1:1000, Sigma) for $5 \mathrm{~min}$. Fluorescent images were captured with a spinning disk confocal microscope (Andor, Belfast, UK).

\section{Protein Extraction and Western Blot}

Cerebral arteries (MCAs, ACAs, and PCAs) were isolated and lysed in RIPA Lysis Buffer (Thermo Scientific, Schwerte, Germany). Total protein was quantified with a Bio-Rad BCA protein assay kit (Pierce Biotechnology, Waltham, MA, USA). Membrane and cytoplasmic proteins were extracted with Membrane and Cytosol Protein Extraction Kits, respectively (Sigma). The percentage of membrane TRPM4 (m) relative to total TRPM4 $(t)$ was calculated. Increase in TRPM4 (m)/TRPM4 (t) ratio was defined as increased TRPM4 translocation [14]. Following electrophoretic gel separation, proteins were moved onto a polyvinylidene fluoride (PVDF) membrane. Optical density of target proteins and internal reference bands were measured by Odyssey Infrared Imaging System (LI-COR Biosciences, Lincoln, NE, USA). Expression was reported as the signal ratio between the target protein and the internal reference from the same lane. Mass fraction of the electrophoretic separation gel was $10 \%$ and that of the concentrated gel was 5\%. Primary antibodies used were polyclonal rabbit anti-TRPM4 (1:50, Abcam) and anti-actin (1:1000, Sigma). Secondary antibody was donkey anti-rabbit (1:100, Abcam).

\section{TRPM4 Macroscopic Current Recording}

Whole-cell patch clamp was utilized to assess TRPM4 macroscopic currents in cerebral artery myocytes using a MultiClamp 700B amplifier (Molecular Devices Corp., San Jose, CA, USA). Recording electrodes (resistance, 3-6 M $\Omega$ ) were pulled from borosilicate glass (outer diameter, $1.5 \mathrm{~mm}$; inner diameter, $1.12 \mathrm{~mm}$; World Precision Instruments, Sarasota, FL, USA). Currents were filtered at $1 \mathrm{kHz}$, digitized at $40 \mathrm{kHz}$, then retained for further evaluation. pClamp 10 Electrophysiology Data Acquisition and Analysis Software (Molecular Devices) were used for data acquisition and analysis, respectively. Recordings were conducted at room temperature [14]. Bathing solution contained (in $\mathrm{mM}$ ) the following: $134 \mathrm{NaCl}, 6 \mathrm{KCl}, 1 \mathrm{MgCl}_{2}, 2 \mathrm{CaCl}_{2}, 10 \mathrm{HEPES}$, and 10 glucose ( $\mathrm{pH}$ 7.4). Pipette solution consisted of $110 \mathrm{mM} \mathrm{K}$ aspartate, $1 \mathrm{mM} \mathrm{MgCl}$, $30 \mathrm{mM} \mathrm{KCl}, 10 \mathrm{mM} \mathrm{NaCl}, 10 \mathrm{mM}$ HEPES, and $5 \mu \mathrm{M}$ EGTA ( $\mathrm{pH}$ 7.2). Osmolarity of the pipette and the bathing solutions was $295 \pm 5$ mOsm, quantified by a vapor pressure osmometer (Wescor Inc., Logan, UT, USA). Current intensity (pA) was recorded with membrane potential $(\mathrm{Vm})$ ranging from -80 to $+50 \mathrm{mV}$ and was normalized to membrane capacitance $(\mathrm{pF})$ and reported as current density $(\mathrm{pA} / \mathrm{pF})[14]$.

\section{Pressurized Myography Study}

Pressurized myography experiments were described in details elsewhere [15]. Briefly, cerebral artery was cannulated on glass micropipettes that were placed in a $5-\mathrm{mL}$ myograph chamber filled with physiological saline solution (PSS) at $37{ }^{\circ} \mathrm{C}$ aerated with $5 \% \mathrm{CO}_{2}, 20 \% \mathrm{O}_{2}$, and $75 \%$ $\mathrm{N}_{2}$. PSS contained (in $\mathrm{mM}$ ) the following: $119 \mathrm{NaCl}, 4.7$ $\mathrm{KCl}, 24 \mathrm{NaHCO}_{3}, 0.2 \mathrm{KH}_{2} \mathrm{PO}_{4}, 1.1$ EDTA, $1.2 \mathrm{MgSO}_{4}$, $1.6 \mathrm{CaCl}_{2}$, and 10.6 glucose ( $\left.\mathrm{pH} 7.4\right)$. Intraluminal pressure was adjusted with a servo-null pressure controller and a peristaltic pump. Variations in arterial diameter were quantified by a video dimension analyzer (Living Systems Instrumentation, St. Albans, VT, USA) and WinDAQ data acquisition software (DATAQ Instruments, Akron, OH, USA). After equilibration for $1 \mathrm{~h}$ at $40 \mathrm{mmHg}$, cerebral artery viability was tested by $60 \mathrm{mM} \mathrm{KCl}$ (PSS isosmotic replacement of $\mathrm{NaCl}$ by $\mathrm{KCl}$ ). Cerebral arteries that had a $<50 \%$ reduction in diameter under these conditions were discarded. After washing out $\mathrm{KCl}$ with PSS, the diameter of cerebral arteries was recorded at $40 \mathrm{mmHg}$ intravascular pressure once stabilized. PSS was then replaced by diltiazem-containing $\mathrm{Ca}^{2+}$-free PSS for the measurement of passive (maximum) diameters.

Myogenic tone was defined as the amount of constriction in cerebral arteries and expressed as a percentage between active and passive arterial diameters at $40 \mathrm{mmHg}$. Myogenic tone due to TRPM4 was defined as the difference in myogenic tone between cerebral arteries obtained from rats treated with 9-Phe versus the saline group [15].

\section{Cortical CBF Measurement}

Rats were anesthetized using the same method described above. Bilateral femoral arteries were exposed for catheterization (24G). Mean arterial pressure (MAP) was continuously monitored in 1 femoral artery catheter. Blood samples were collected from another femoral artery catheter for blood gas analysis. A hole (1.5 $\mathrm{mm}$ in diameter) was drilled on the skull at $5 \mathrm{~mm}$ lateral and $3 \mathrm{~mm}$ posterior to the bregma. A laser Doppler probe (PF5000, PERIMED, Jarfalla, Sweden) was placed on top of the dura [16]. Cortical CBF was continuously measured for $15 \mathrm{~min}$ after 15 -min equilibration. Cortical $\mathrm{CBF}$ was expressed in perfusion units (PU), which was a unified form after computerized standardization [17]. 


\section{Global CBF Measurement}

After cortical CBF measurement, global CBF measurement was measured by fluorescent microspheres as described elsewhere [6]. Briefly, reference blood samples were collected by a $24 \mathrm{G}$ catheter connected to the right femoral artery. To infuse microspheres, a 26G catheter was percutaneously inserted into the left cardiac ventricle at about $1.5 \mathrm{~cm}$ superior to the ensiform process, adjacent to the left border of the sternum. Catheters were filled with $0.9 \%$ saline supplemented with $20 \mathrm{U} / \mathrm{mL}$ heparin before use. Three milliliters of $0.9 \%$ saline that contained about 300,000 15- $\mu \mathrm{m}$-diameter fluorescent microspheres (Invitrogen, Carlsbad, CA, USA) was administered at a rate of $1.0 \mathrm{~mL} / \mathrm{min}$. In addition, reference arterial blood sample with injected microspheres was simultaneously collected at a rate of $1.3 \mathrm{~mL} / \mathrm{min}$. Brains were collected postmortem. Collected brain tissues and reference blood samples were digested in ethanolic potassium hydroxide to recover microspheres. Microspheres isolated from each specimen were dissolved in 2-ethoxyethyl acetate. Dissolved fluorescent dyes were then analyzed by a fluorometer (Model 814, Photon Technology International, Birmingham, NJ, USA). CBF was estimated with the following formula: $\mathrm{CBF}[\mathrm{mL} /$ $(100 \mathrm{~g}$ min $)]=[F$ (brain) $/ F$ (Reference) $\times$ Rate $(\mathrm{mL} / \mathrm{min})] /$ Weight $(\mathrm{g})$, in which " $F$ (brain)" referred to brain sample fluorescence intensity, " $F$ (Reference)" referred to reference blood sample fluorescence intensity, "Rate" is the reference blood sample withdrawal rate, and "Weight" is the weight of the brain [11].

\section{Data Analysis and Statistics}

The statistical data were assessed using the Hartley test for homogeneity of variance, and the measurement data conforming to the normal distribution were expressed as mean \pm standard deviation (S.D.). Data between groups were compared by two-way ANOVA and Dunnett's post hoc test. Differences were considered significant when $p<0.05$.

\section{Results}

\section{TRPM4 Expression and Membrane Translocation Are Increased in Cerebral Artery Myocytes After SAH}

TRPM4 was expressed in cerebral artery myocytes from both the sham (Fig. 3a-c) and SAH (Fig. 3d-f) groups. Representative TRPM4 protein bands are shown in Fig. 3g. Total TRPM4 protein expression in cerebral artery myocytes was higher in the SAH group compared to the sham group at day $3(31.1 \pm 2.3 \%$
Fig. 3 TRPM4 protein expression and membrane translocation in cerebral artery myocytes. Representative immunofluorescence images at day 5 (a-f). $\alpha$-Actin (green) is a specific marker for myocytes. Representative Western blot protein bands at day 5 for total TRPM4 (t), membrane TRPM4 (m), and cytoplasmic TRPM4 (c) (g). TRPM4 protein expression in cerebral artery myocytes in sham and SAH groups at day 3 , day 5 , and day 7 (h). TRPM4 membrane translocation in cerebral artery myocytes in sham and SAH groups at day 3 , day 5 , and day 7 (I). $* p<0.05, * * p<0.01$
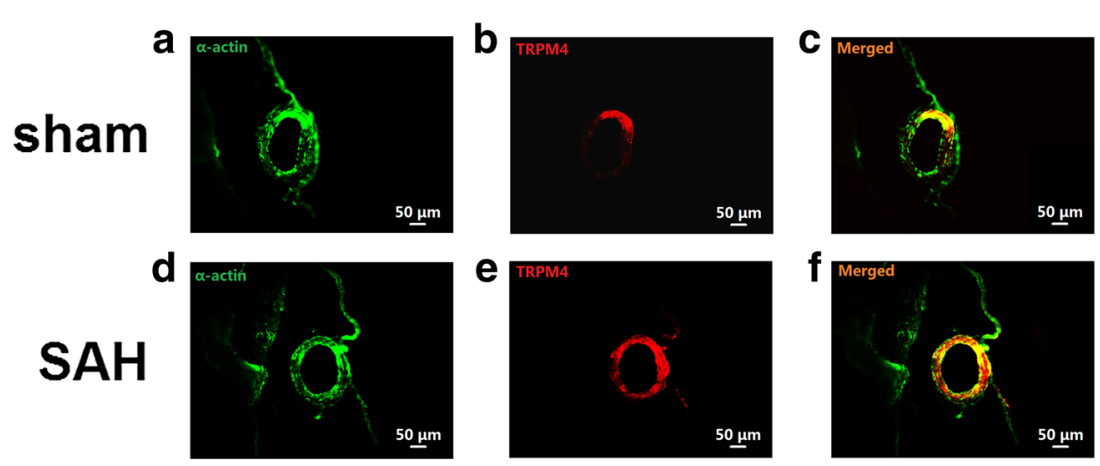

g
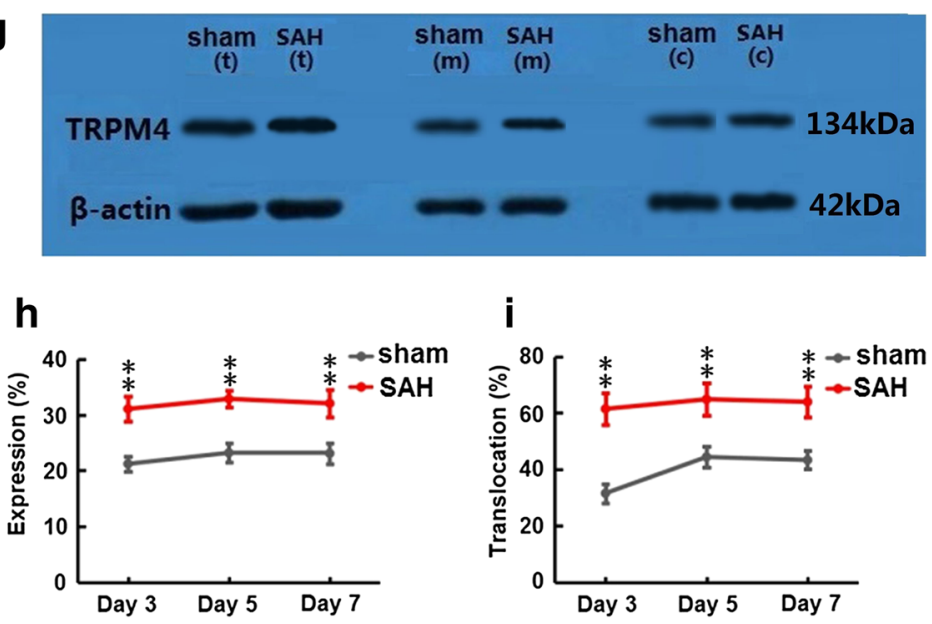
vs. $21.2 \pm 1.4 \%, p<0.01)$, day $5(32.9 \pm 1.5 \%$ vs. $23.2 \pm 1.7 \%$, $p<0.01)$, and day $7(32.1 \pm 2.5 \%$ vs. $23.1 \pm 1.9 \%, p<0.01)$ (Fig. $3 \mathrm{~h})$. TRPM4 membrane translocation in cerebral artery myocytes was also greater in the $\mathrm{SAH}$ group relative to the sham group at day $3(61.4 \pm 3.0 \%$ vs. $31.4 \pm 2.5 \%, p<0.01)$, day $5(64.8 \pm$ $4.0 \%$ vs. $44.3 \pm 2.4 \%, p<0.01)$, and day $7(63.9 \pm 3.6 \%$ vs. $43.2 \pm 2.6 \%, p<0.01$ ) (Fig. 3i).

\section{TRPM4 Macroscopic Currents in Cerebral Artery Myocytes Are Increased After SAH}

TRPM4 macroscopic current density in cerebral artery myocytes (Table 1) was increased in the SAH group compared to the sham group at day 3 (Fig. 4c), day 5 (Fig. 4g), and day 7 (Fig. 4k). Representative tracings show that 9-Phe almost completely inhibited macroscopic currents at day 5 in both the sham (Fig. 4a) and SAH (Fig. 4b) groups. Summary graphs also show that 9-Phe significantly decreased macroscopic currents at day 3 (Fig. 4d), day 5 (Fig. 4h), and day 7 (Fig. 4l) in the sham and SAH groups (Fig. 4e, i, m). However, there was no significant difference in TRPM4 macroscopic current density between the 9-Phe-treated sham and SAH groups at day 3 (Fig. 4f), day 5 (Fig. 4j), and day 7 (Fig. 4n).

\section{TRPM4 Mediates Increased Myogenic Tone in Cerebral Arteries After SAH}

Representative tracings show that cerebral arteries dilated to 9Phe and further dilated in calcium-free PSS in the sham group at day 3 (Fig. 5a), day 5 (Fig. 5c), and day 7 (Fig. 5e). Similar results were also found in the SAH group at day 3 (Fig. 5b), day 5 (Fig. 5d), and day 7 (Fig. 5f). Myogenic tone in cerebral arteries (Table 2) was higher in the SAH group compared to the sham group at day $3(p<0.05)$, day $5(p<0.05)$, and day 7 $(p<0.05)$ (Fig. 5g). Treatment with 9-Phe significantly reduced myogenic tone in cerebral arteries in both the sham (Fig. 5h) and SAH (Fig. 5i) groups at day $3(p<0.01)$, day $5(p<0.01)$, and day $7(p<0.01)$. Myogenic tone was not different between the 9-Phe-treated sham and SAH groups at day 3, day 5, and day 7 (Fig. 5j). Furthermore, magnitude of reduction in myogenic tone by 9-Phe was higher in the SAH group compared to the sham group at day $3(p<0.05)$, day $5(p<0.05)$, and day 7 $(p<0.05)$ (Fig. 5k).

\section{TRPM4 Contributes to CBF Reduction After SAH}

MAP, arterial blood $\mathrm{PaO}_{2}, \mathrm{PaCO}_{2}$, and $\mathrm{pH}$ were in physiological ranges and not different among groups during $\mathrm{CBF}$ measurements at day 3, day 5, and day 7 (Table 3). Cortical (Fig. 6a) and global (Fig. 6e) CBFs were lower in the $\mathrm{SAH}$ group than in the sham group at day $3(p<0.01)$, day $5(p<0.01)$, and day 7 $(p<0.01)$. Treatment with 9-Phe improved cortical (Fig. 6b) and global (Fig. 6f) CBFs in the sham group at day $3(p<0.01)$, day 5

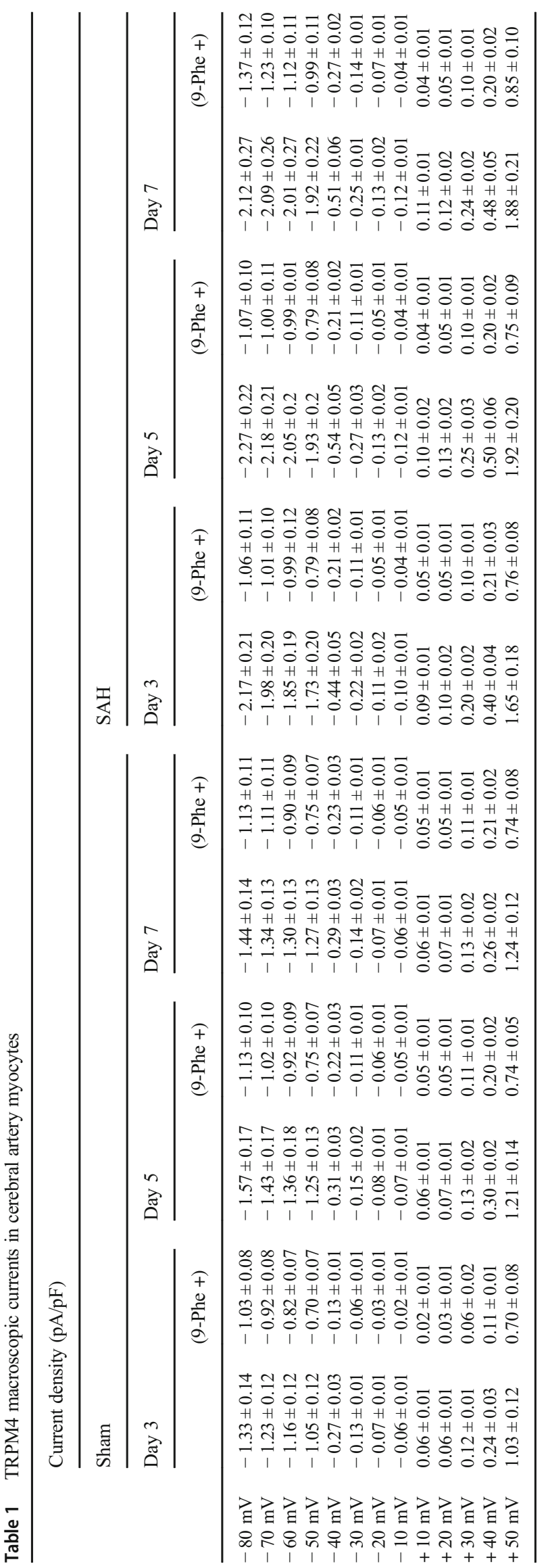


a

Day 5

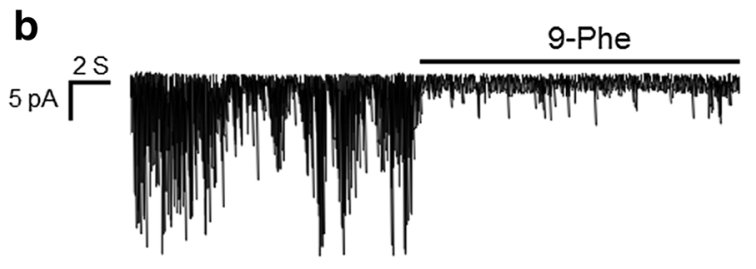

sham $(\mathrm{Vm}=-80 \mathrm{mV})$

$\mathrm{SAH}(\mathrm{Vm}=-80 \mathrm{mV})$

C

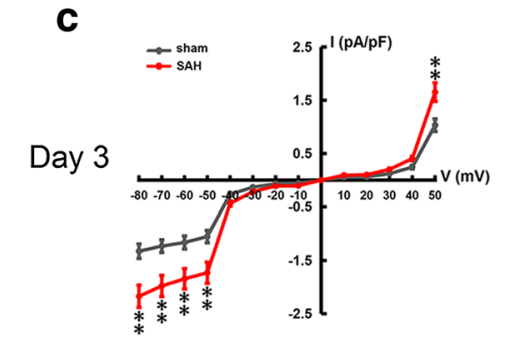

d
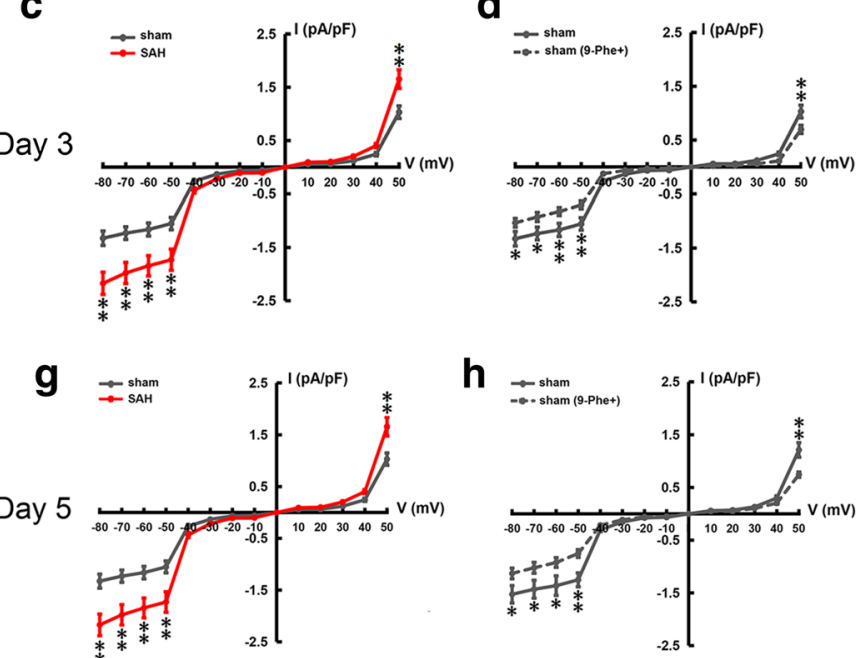

h

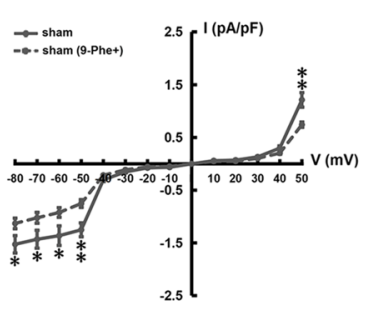

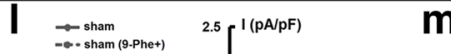

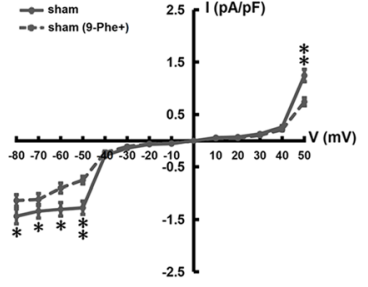

Fig. 4 TRPM4 macroscopic currents recorded in cerebral artery myocytes. TRPM4 current traces recorded by whole-cell patch clamp $(\mathrm{Vm}=-80 \mathrm{mV})$ in sham and SAH groups at day 5. TRPM4 current traces were recorded in bathing solution as a baseline and then with

e

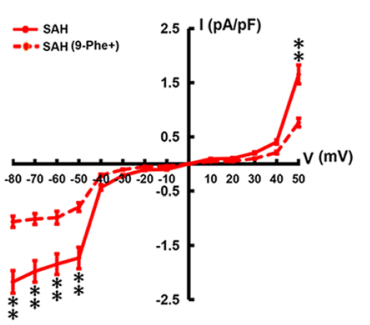

i

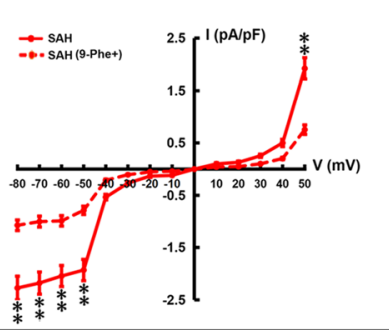

$\mathbf{m}_{\substack{\rightarrow \rightarrow \text { SAAH } \\ \rightarrow \text { SAPhe+) }}}$

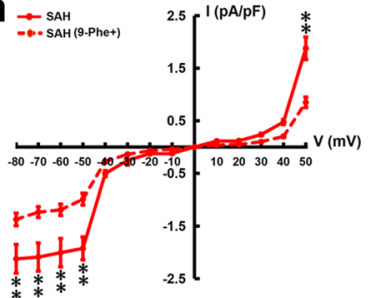

f

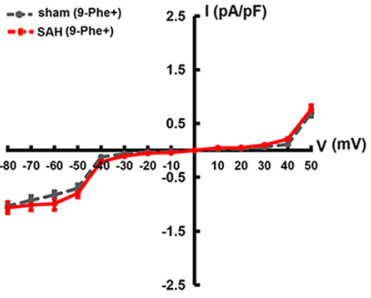

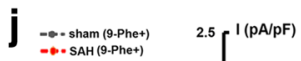

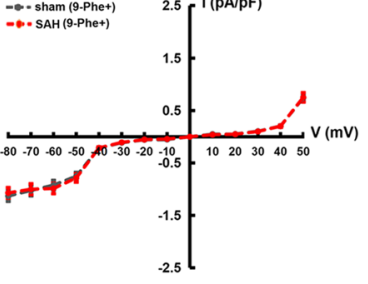

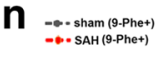

$2.55^{1(\mathrm{PA} / \mathrm{PF})}$

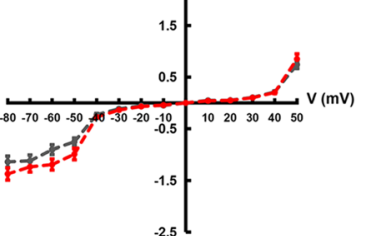

addition of $0.03 \mathrm{mM}$ 9-Phe (a, b). Comparison of TRPM4 macroscopic current density between different groups at day $3(\mathbf{c}-\mathbf{f})$, day $5(\mathbf{g}-\mathbf{j})$, and day 7 (k-n). $* p<0.05, * * p<0.01$

macroscopic current density was increased after SAH; (3) TRPM4 accounted for the increased proportion of myogenic tone after SAH; and (4) TRPM4 contributed to CBF reduction after SAH. These findings collectively provided strong evidence that increased TRPM4 activity in cerebral artery myocytes contributes to $\mathrm{CBF}$ reduction after SAH.

Launay et al. [9] first reported the amino acid sequence of TRPM4. Subsequently, a series of studies established that TRPM4 participates in various physiological processes [18]. For example, TRPM4 regulates cytokine secretion in lymphocytes as well as insulin secretion in pancreatic $\beta$-cells [19]. TRPM4 also participates in the control of respiratory rhythmogenesis involving neurons of the pre-Bötzinger complex [20] and has been implicated in the degeneration of axons and neurons in experimental multiple sclerosis [18]. In terms

In this study, we examined TRPM4 activity and expression in cerebral artery myocytes and their contribution to CBF reduction after SAH. Our results demonstrated that (1) TRPM4 expression and translocation were increased after SAH; (2) TRPM4 

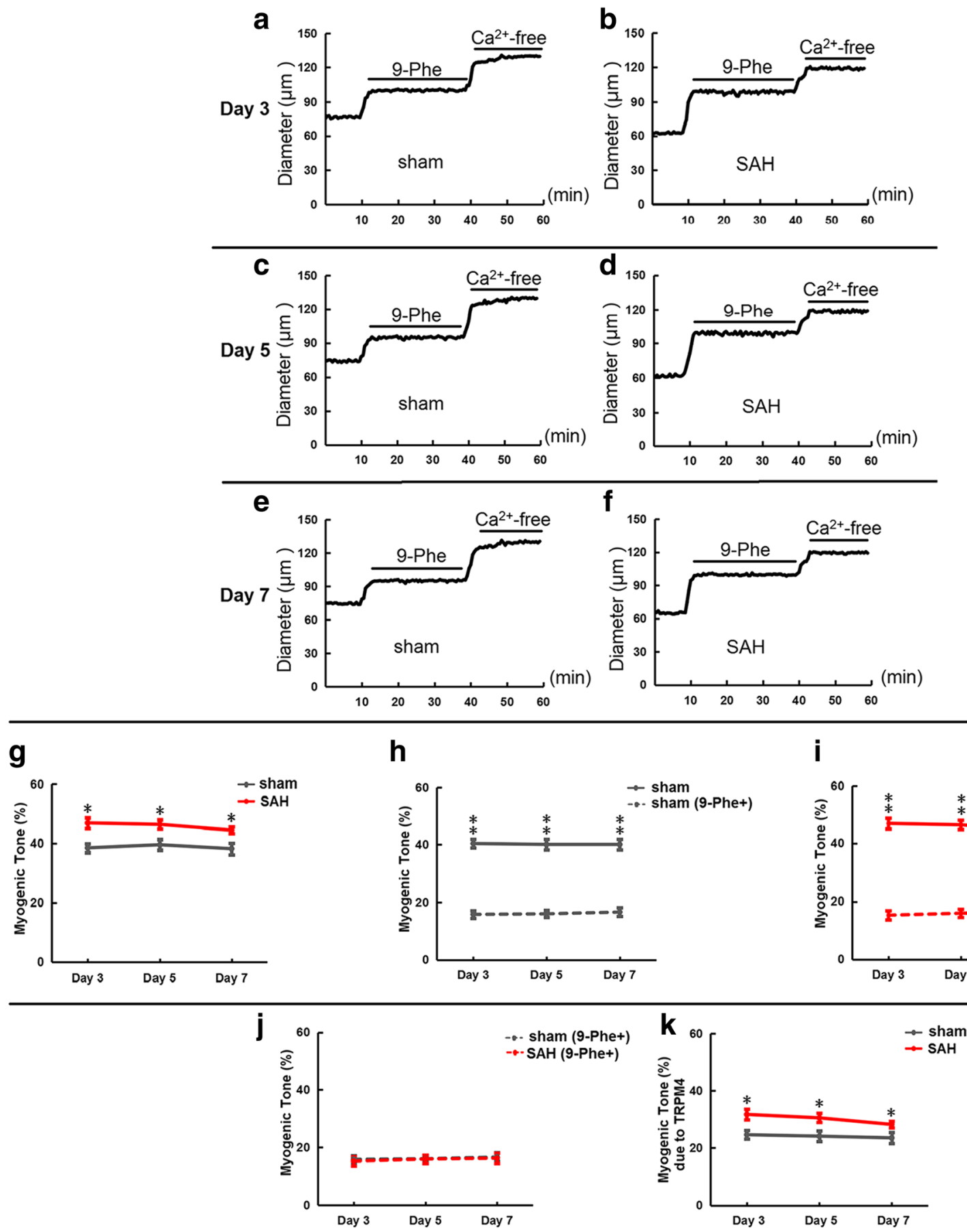

Fig. 5 Pressurized myography traces and myogenic tone of cerebral arteries in response to 9-Phe. Representative tracings showing cerebral artery diameter in different groups at day 3 , day 5 , and day 7 (intraluminal pressure $=40 \mathrm{mmHg})(\mathbf{a}-\mathbf{f})$. Comparison of myogenic tone between

different groups at day 3 , day 5 , and day $7(\mathbf{g}-\mathbf{j})$. Myogenic tone due to TRPM4 in sham and SAH groups at day 3 , day 5 , and day $7(\mathbf{k})$. $* p<0.05, * * p<0.01$

of the heart, TRPM4 has been associated with early afterdepolarizations related to reoxygenation after hypoxia [21]. Gain-of-function point mutations in the TRPM4 gene have been implicated to a genetic form of conduction blockage of the heart, suggesting that the channel portrays a role in cardiac Purkinje fibers [22, 23].

Several studies have described the detection of TRPM4 mRNA in native cerebral artery myocytes and indicated that TRPM4 is critically important for pressure-induced myocyte depolarization, cerebral artery myogenic tone, and CBF regulation [10]. Importantly, TRPM4 is mechanosensitive and therefore senses and reacts to blood pressure changes and other 
Table 2 Cerebral artery myogenic tone

Myogenic tone (\%)

\begin{tabular}{llll}
\hline & Day 3 & Day 5 & Day 7 \\
\hline Sham & $40.4 \pm 1.5$ & $40.1 \pm 1.8$ & $40.1 \pm 1.9$ \\
Sham (9-Phe +) & $15.8 \pm 1.3$ & $16.0 \pm 1.2$ & $16.6 \pm 1.5$ \\
Due to TRPM4 (sham) & $24.6 \pm 1.5$ & $24.1 \pm 1.8$ & $23.5 \pm 1.9$ \\
SAH & $46.9 \pm 1.8$ & $46.4 \pm 1.6$ & $44.4 \pm 1.2$ \\
SAH (9-Phe +) & $15.2 \pm 1.6$ & $15.9 \pm 1.4$ & $16.2 \pm 1.8$ \\
Due to TRPM4 (SAH) & $31.7 \pm 1.8$ & $30.5 \pm 1.6$ & $28.2 \pm 1.2$ \\
\hline
\end{tabular}

types of mechanical stimulation, converting extracellular physical signals to intracellular signals that cause changes in cerebral artery myocyte contractility and hence myogenic tone [9]. CBF regulation involves the integration of data derived from 3 major signaling inputs using myogenic, metabolic, and neurogenic mechanisms. Cerebral artery myogenic tone has a crucial role in regulating $\mathrm{CBF}[24,25]$. Earley et al. [26] documented that administration of a TRPM4 pharmacological inhibitor to isolated rat cerebral arteries exhibiting pre-developed myogenic tone led to cerebral artery myocyte hyperpolarization and relaxation of the cerebral arteries to nearly passive diameter, indicating that TRPM4 participates in pressure-dependent depolarization as well as in the control of cerebral artery myogenic tone. Reading and Brayden [11] revealed that introduction of TRPM4 antisense oligonucleotides into the cerebral spinal fluid of rats via the third cerebral ventricle results in the downregulation of TRPM4 in cerebral artery myocytes, which in turn significantly increased CBF, suggesting that their cerebral arteries did not appropriately react to alterations in perfusion pressure. These data provide strong evidence that TRPM4 has a pivotal role in the proper regulation of cerebral artery myogenic tone. Thus, TRPM4 is involved in the regulation of cerebral artery function and $\mathrm{CBF}$ under physiological conditions.

In addition to showing an increase in TRPM4 activity after $\mathrm{SAH}$, this study also demonstrates that inhibition of TRPM4 activity after SAH led to decreased cerebral artery myogenic tone and significant CBF recovery, suggesting TRPM4 has a pivotal role in $\mathrm{CBF}$ reduction after $\mathrm{SAH}$. It has been hypothesized that myogenic tone has a primary role in regulating blood flow in various organ systems, particularly the kidney and brain [24]. We conclude that TRPM4 activity is essential for the development as well as preservation of myogenic tone in the cerebral arteries and that TRPM4 is the main regulator of cerebral artery function. Cerebral artery myogenic reactivity to changes in intraluminal pressure is a major contributor to the mechanism of CBF regulation and is reduced in the presence of TRPM4 inhibition and is the first to demonstrate a role for a mechanosensitive ion channel in $\mathrm{CBF}$ regulation. The cause of $\mathrm{CBF}$ reduction after $\mathrm{SAH}$ is multifactorial and complicated [27]. Factors including cerebral artery endothelial dysfunction, sympathetic neurological dysfunction, cerebral arterial wall inflammatory responses, and cerebral artery microthrombosis are involved in CBF reduction. Thus, CBF reduction after SAH likely involves other pathophysiological mechanisms in addition to changes in TRPM4 activity.

This study demonstrates that TRPM4 expression, translocation, and macroscopic current intensity were increased after SAH, suggesting that SAH has an induction effect on TRPM4 activity and that they are closely related. A molecular mechanism of SAH-mediated upregulation of TRPM4 expression and activity has not been reported. SAH has been shown to induce upregulation of dozens of ion-gated or chemically gated channels, but related signaling pathways have not been elucidated. Importantly, properties of TRPM4 such as $\mathrm{Ca}^{2+}$ dependence and sensitivity to PKC activity may be involved [28]. TRPM4 activation after SAH may function as an integrator of PKC activity as well as intracellular $\mathrm{Ca}^{2+}$ to generate a physiological reaction in cerebral artery myocytes. Thus, the control of TRPM4 activity by PKC mainly controls cerebral artery myogenic tone after $\mathrm{SAH}$ and in the regulation of $\mathrm{CBF}$ [29]. Previous studies have confirmed that PKC activity was increased after SAH [30]. PKC activation has been shown to improve TRPM4 sensitivity to intracellular $\mathrm{Ca}^{2+}$ [29], inducing TRPM4 activation and the generation of a large amount of inward currents. Enhanced inward currents lead to depolarization of cerebral artery myocytes and activation of voltagedependent calcium channels (VDCCs), resulting in massive $\mathrm{Ca}^{2+}$ influx and cerebral artery contraction [30]. Additionally, PKC increases VDCC activity directly [31]. The current study confirms that $\mathrm{Ca}^{2+}$ influx-dependent mechanisms are largely responsible for cerebral artery constriction and maintenance of cerebral artery myogenic tone [29]. Increases in cerebral artery pressure and $\mathrm{Ca}^{2+}$ influx may further activate TRPM4, resulting in increased TRPM4 activity after SAH.
Table 3 Arterial MAP, $\mathrm{PaO}_{2}$, $\mathrm{PaCO}_{2}$, and $\mathrm{pH}$

\begin{tabular}{llllll}
\hline & $\mathrm{n}$ & $\mathrm{MAP}(\mathrm{mmHg})$ & $\mathrm{PaO}_{2}(\mathrm{mmHg})$ & $\mathrm{PaCO}_{2}(\mathrm{mmHg})$ & $\mathrm{pH}$ \\
\hline Sham & 18 & $105 \pm 5$ & $166 \pm 13$ & $34 \pm 3$ & $7.42 \pm 0.02$ \\
SAH & 18 & $109 \pm 3$ & $173 \pm 10$ & $35 \pm 2$ & $7.41 \pm 0.02$ \\
Sham + 9-Phe & 18 & $104 \pm 3$ & $170 \pm 11$ & $34 \pm 2$ & $7.39 \pm 0.01$ \\
SAH + 9-Phe & 18 & $107 \pm 3$ & $169 \pm 10$ & $34 \pm 3$ & $7.39 \pm 0.02$ \\
\hline
\end{tabular}

$\mathrm{MAP}=$ mean arterial blood pressure $\mathrm{n}=$ number of animals; $\mathrm{PaO}_{2}=$ arterial oxygen partial pressure; $\mathrm{PaCO}_{2}=$ carbon dioxide partial pressure 
a

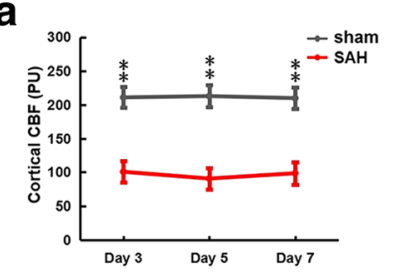

b

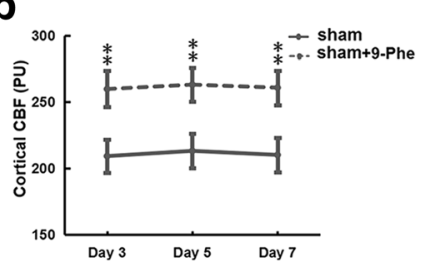

C

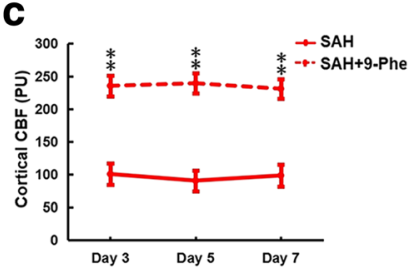

d

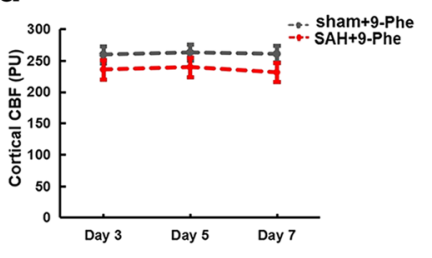

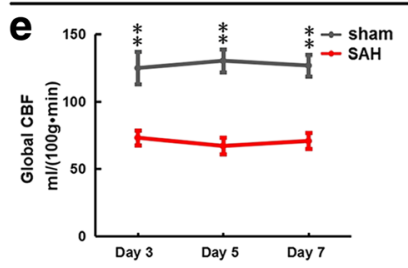
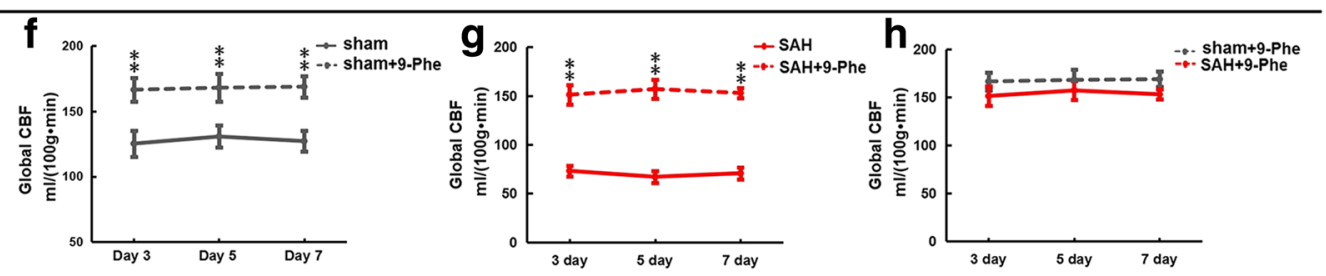

Fig. 6 Cortical and global CBF. Comparison of cortical (a-d) and global (e-h) CBFs between different groups at day 3, day 5, and day 7. *p<0.05, $* * p<0.01$

An effect of PKC on TRPM4 activity may also be reflected by our data regarding TRPM4 translocation [14]. TRPM4 is expressed in both cell membrane and cytoplasm in cerebral artery myocytes, but only TRPM4 that localized at cell membrane has ion channel function [14]. Increased PKC activity after SAH induces acute TRPM4 trafficking to the cell membrane, causing a fast elevation in membrane TRPM4 levels in cerebral artery myocytes, which increases the number of channels that are activated by intracellular $\mathrm{Ca}^{2+}$, and thus the sensitivity of cerebral artery myocytes to intraluminal pressure changes [14]. Changes in TRPM4 levels at the cerebral artery myocyte membrane by PKC may thus be a pivotal element in the control of cerebral artery myocyte membrane potential and cerebral artery myogenic tone.

Table 4 Cortical CBF

Cortical CBF (PU)

\begin{tabular}{llll}
\hline & Day 3 & Day 5 & Day 7 \\
\hline Sham & $209.14 \pm 15.44$ & $213.1 \pm 16.06$ & $210.05 \pm 15.97$ \\
SAH & $100.64 \pm 16.01$ & $90.53 \pm 15.83$ & $98.43 \pm 16.72$ \\
Sham + 9-Phe & $259.89 \pm 13.72$ & $263.22 \pm 12.72$ & $260.92 \pm 13.01$ \\
SAH + 9-Phe & $235.96 \pm 15.72$ & $239.86 \pm 15.72$ & $231.45 \pm 15.01$ \\
\hline
\end{tabular}

$\mathrm{PU}=$ perfusion unit for cerebral blood flow

Table 5 Global CBF

Global CBF $\mathrm{mL} /(100 \mathrm{~g} \min )$

\begin{tabular}{llll}
\hline & Day 3 & Day 5 & Day 7 \\
\hline Sham & $125.23 \pm 12.14$ & $130.66 \pm 8.48$ & $127.1 \pm 7.97$ \\
SAH & $73.25 \pm 5.39$ & $67.15 \pm 6.25$ & $70.83 \pm 5.98$ \\
Sham + 9-Phe & $166.66 \pm 9.11$ & $166.66 \pm 9.11$ & $169.01 \pm 8.19$ \\
SAH + 9-Phe & $151.45 \pm 10.01$ & $157.25 \pm 9.76$ & $153.22 \pm 5.43$ \\
\hline
\end{tabular}

An increase in TRPM4 activity after SAH may also result from interactions with other TRP channels. A critical role for TRPC6 in cerebral artery myogenic constriction has been elucidated [32]. TRPC6 is a $\mathrm{Ca}^{2+}$-permeable mechanosensitive ion channel [32]. The fact that inhibition of either TRPC6 or TRPM4 results in comparable suppression of pressure-induced depolarization and myogenic tone implies that these 2 TRP channels engage in cooperative interactions [10]. Previous studies have suggested that mechanical stress stimulates TRPC6, which leads to $\mathrm{Ca}^{2+}$ entry and activation of nearby TRPM4. $\mathrm{Na}^{+}$entry via TRPM4 subsequently depolarizes cerebral artery myocytes and induces cerebral artery contraction and CBF reduction [10]. Validation of this suggested TRP channel interaction is warranted. Studies of this type will provide important insights about the integrated function and diversity of the physiological roles of TRP channels in cerebral artery myocytes.

In a previous study, we found the role of R-type calcium channels in relation to $\mathrm{CBF}$ regulation after SAH [7], but the upstream mechanism is still unknown. This study elucidates the role of a mechanosensitive ion channel in the control of cerebral artery function and suggests an important functional role for TRPM4 in cerebral artery myocytes. This implicates TRPM4 as a major contributor to a reduction in CBF in vivo after SAH. TRPM4 may serve as an upstream mediator for the effect of R-type calcium channels on the regulation of CBF after SAH. This study identifies a new target, TRPM4, for the study of cerebral vasospasm, and also for the development of novel clinical interventions to treat cerebral vasospasm.

Acknowledgments The authors thank Dr. Siu-Lung Chan for critically review and intellectual input on this manuscript. This work was supported by the National Natural Science Foundation of China (Nos. 81760223, 81560206), Natural Science Foundation of Yunnan Province (Nos. FB2016121, 2014FB087), Yunnan Health Training Project in High Level Talents (No. H-201601), Technology and Science Innovation Team Foundation of Kunming Medical University (No. CXTD201707), Yunnan Key Laboratory of Medicine Funding (No. 2017DG005), and Internal Funding of Yunnan Provincial Health and Family Planning Commission (No. 2016NS205). 


\section{Compliance with Ethical Standards}

Conflict of Interest The authors declare that the research was conducted in the absence of any commercial or financial relationships that could be construed as a potential conflict of interest.

Required Author Forms Disclosure forms provided by the authors are available with the online version of this article.

\section{References}

1. Dasenbrock HH, Angriman F, Smith TR, et al. Readmission after aneurysmal subarachnoid hemorrhage: a nationwide readmission database analysis. Stroke 2017; 48(9): 2383-2390.

2. Kang DW. Intracerebral hemorrhage: large disease burden but less therapeutic progress. J Stroke 2017; 19(1): 1-2.

3. Rostami E, Engquist H, Johnson U, et al. Monitoring of cerebral blood flow and metabolism bedside in patients with subarachnoid hemorrhage - A Xenon-CT and microdialysis study. Front Neurol 2014; 5: 89.

4. Tso MK, Macdonald RL. Subarachnoid hemorrhage: a review of experimental studies on the microcirculation and the neurovascular unit. Transl Stroke Res 2014; 5(2): 174-189.

5. Bauer AM, Rasmussen PA. Treatment of intracranial vasospasm following subarachnoid hemorrhage. Front Neurol 2014; 5: 72.

6. Wang F, Yin YH, Jia F, Jiang JY. Antagonism of R-type calcium channels significantly improves cerebral blood flow after subarachnoid hemorrhage in rats. J Neurotraum 2010; 27(9): 1723-1732.

7. Szarka N, Amrein K, Horvath P, et al. Hypertension-induced enhanced myogenic constriction of cerebral arteries is preserved after traumatic brain injury. J Neurotraumal 2017; 34(14): 2315-2319.

8. Simms BA, Zamponi GW. Neuronal voltage-gated calcium channels: structure, function, and dysfunction. Neuron 2014; 82(1): 24 45.

9. Guo J, She J, Zeng W, Chen Q, Bai XC, Jiang Y. Structures of the calcium-activated, non-selective cation channel TRPM4. Nature 2017; 552(7684): 205-209.

10. Earley S, Waldron BJ, Brayden JE. Critical role for transient receptor potential channel TRPM4 in myogenic constriction of cerebral arteries. Circ Res 2004; 95(9): 922-929.

11. Reading SA, Brayden JE. Central role of TRPM4 channels in cerebral blood flow regulation. Stroke 2007; 38(8): 2322-2328.

12. Ding XQ, Ban T, Liu ZY, et al. Transient receptor potential melastatin 4 (TRPM4) contributes to high salt diet-mediated early-stage endothelial injury. Cell Physiol Biochem 2017; 41(2): 835-848.

13. Wang F, Koide M, Wellman GC. Nifedipine inhibition of highvoltage activated calcium channel currents in cerebral artery myocytes is influenced by extracellular divalent cations. Front Physiol 2017; 8: 210-220.

14. Crnich R, Amberg GC, Leo MD, et al. Vasoconstriction resulting from dynamic membrane trafficking of TRPM4 in vascular smooth muscle cells. Am J Physiol Cell Physiol 2010; 299(3): C682-694.

15. Syed AU, Koide M, Brayden JE, Wellman GC. Tonic regulation of middle meningeal artery diameter by ATP-sensitive potassium channels. J Cerebr Blood F Met 2017; 1: $271678 X 17749392$.
16. Kubasch ML, Kubasch AS, Torres Pacheco J, et al. Laser doppler assessment of vasomotor axon reflex responsiveness to evaluate neurovascular function. Front Neurol 2017; 8: 370.

17. Tabrizchi R, Pvugsley MK. Methods of blood flow measurement in the arterial circulatory system. J Pharmacol Toxicol Methods 2000; 44(2): 375-384.

18. Bianchi B, Smith PA, Abriel H. The ion channel TRPM4 in murine experimental autoimmune encephalomyelitis and in a model of glutamate-induced neuronal degeneration. Mol Brain 2018; 11(1): 41.

19. Ma Z, Björklund A, Islam MS. A TRPM4 inhibitor 9-Phenanthrol inhibits glucose-and glucagon-like peptide 1-induced insulin secretion from rat islets of langerhans. J Diabetes Res 2017; 2017: 5131785.

20. Mironov SL. Calmodulin and CaMKII mediate emergent bursting activity in the brainstem respiratory network (preBötzinger complex). J Physiol 2013; 591(7): 1613-1630.

21. Wang C, Naruse K, Takahashi K. Role of the TRPM4 channel in cardiovascular physiology and pathophysiology. Cells 2018; 7(6): 62.

22. Liu H, El Zein L, Kruse M, et al. Gain-of-function mutations in TRPM4 cause autosomal dominant isolated cardiac conduction disease. Circ Cardiovasc Genet 2010; 3(4): 374-385.

23. Tian J, An XJ, Fu MY. Transient receptor potential melastatin 4 cation channel in pediatric heart block. Eur Rev Med Pharmacol Sci 2017; 21(4 Suppl): 79-84.

24. Palomares SM, Cipolla MJ. Myogenic tone as a therapeutic target for ischemic stroke. Curr Vasc Pharmacol 2014; 12(6): 788-800.

25. Ter Laan M, Van Dijk JM, Elting JW, Staal MJ, Absalom AR. Sympathetic regulation of cerebral blood flow in humans: a review. Br J Anaesth 2013; 111(3): 361-367.

26. Gonzales AL, Garcia ZI, Amberg GC, Earley S. Pharmacological inhibition of TRPM4 hyperpolarizes vascular smooth muscle. Am J Physiol Cell Physiol 2010; 299(5): C1195-1202.

27. An SJ, Kim TJ, Yoon BW. Epidemiology, risk factors, and clinical features of intracerebral hemorrhage: an update. J Stroke 2017; 19(1): 3-10.

28. Earley S. TRPM4 channels in smooth muscle function. Pflugers Arch 2013; 465(9): 1223-1231.

29. Earley S, Straub SV, Brayden JE. Protein kinase C regulates vascular myogenic tone through activation of TRPM4. Am J Physiol Heart Circ Physiol 2007; 292(6): H2613-2622.

30. Wang F, Wang Y, Xiao XF, Wang HZ, Sun T, Yu HL. Effect of arterial and venous subarachnoid hemorrhage on voltage-dependent calcium channel currents of cerebral artery smooth muscle cells. Chin J Cerebrovac Dis 2015; 12(2): 78-82.

31. Tsouka V, Markou T, Lazou A. Differential effect of ischemic and pharmacological preconditioning on PKC isoform translocation in adult rat cardiac myocytes. Cell Physiol Biochem 2002; 12(5-6): 315-324.

32. Zeng C, Tian F, Xiao B. TRPC channels: prominent candidates of underlying mechanism in neuropsychiatric diseases. Mol Neurobiol 2016; 53(1): 631-647.

Publisher's Note Springer Nature remains neutral with regard to jurisdictional claims in published maps and institutional affiliations. 